\title{
The Optimal Positioning Algorithm Based on RSSI of WiFi
}

\author{
Haibo Liu, Yujie Dong and Yongle Ai \\ School of Electric Engineering and Automation, Henan Polytechnic University. \\ liuhaibo09@hpu.edu.cn
}

\begin{abstract}
The positioning system based on RSSI is extremely affected by the multipath transmission medium, the signal transmission and shadow. Even changing the angle of the antenna is likely to affect the ranging accuracy. In order to solve the problems above, this paper proposes an indoor optimal positioning algorithm based on Wi-Fi signat strength. It introduces positioning evaluation function, using the optimal algorithm to get a set of path loss exponent to improve the positioning accuracy. Experimen results show that the algorithm can obtain higher positioning accuracy without priopi information of indoor environment or additional hardware investment.
\end{abstract}

Keywords: WiFi; RSSI; Distance Loss Model; OptimalPositioning

\section{Introduction}

The technology of Wi-Fi is widely exploited in nowadays network. It has been a part of people's work, study and entertainmentlife, which ponides convenient and high quality network service for people. At the same time the seamless Location Based Services (LBS) attracts the attention of most users. Therefore, the location technology based on Wi-Fi rapidly arouses people's much attention, and quickly becomes the focus of the indoor location technology research due toits advantages of low cost of deployment, easy to implement, high positiongng accuract and easy extension.

The current mainstream of indoor localization algorithm is mainly based on RSSI, which is with no need for additional hardware investment. There are a large number of Wi-Fi nodes in our life, as long as prior to get name and coordinates of locating wireless AP, it can be used as pontroning AP nodes. Positioning algorithm based on RSSI is divided into two categories. non ranging algorithm and ranging algorithm. At present, many scholars have made a lot of exploration and research on the localization method based on the path-loss model. In the positioning process, the main problem is that the wireless signal is influenced by the positioning environment obvious, and the influence of different obstacles on the wireless signal cannot be predicted. In the literature [2, 3], in order to eliminate the influence of indoor positioning environment on the positioning accurac the secondary positioning algorithm based on RSSI is proposed. First according to the existing network information, the location terminal is roughly located, and then acco ding to a rough estimate of the location update path loss index, and then second times to locate, to improve the positioning effect. In the literature [4], the secondary positioning method is adopted to obtain the better positioning effect by modifying the path loss exponent in the indoor environment. Based on the existing algorithms, this paper proposes an optimal positioning algorithm based on RSSI, by introducing the positioning evaluation function, the optimization algorithm is used to obtain a set of optimal path loss index to improve the positioning accuracy in the presence of non line of sight error. 


\section{RSSI Data Processing}

Indoor environment is complex and changeable, due to wave signal of non line of sight propagation, multipath propagation, and other electronic devices in the signal to interference, the intensity fluctuation for receiving RSSI is bigger, the value of great uncertainty and randomness. Before the system is located, the measured signal strength must be processed by filtering, so that the measured value is close to the true value.

\subsection{Data Error Processing}

The error of RSSI in the measurement process can be divided into random error, system error and gross error. When the transmitter and the receiver does not exist non line-of-sight error, the fluctuation amplitude of RSSI is small, which is mainly due to the random error and the gross error of the part. On the contrary, the measurement result is violent fluctuation, gross error and random error are very obvious, it is need to remoye part of the existence of coarse error before positioning, and then make the filter to avoid the influence of the positioning accuracy of the system.

(1) Gross error elimination

At present, there are many ways to achieve eliminate gross etrors in the data, the simplest is repeated measurements, Pauta criterion and Grubbs criterion is the most commonly used mathematical methods. According to the requirements for indoor positioning and feature of Wi-Fi signal intensity, this paper selects Pauta test to eliminate gross error. If a RSSI measurement value and all measured values of average value of the difference in the absolute value is greater than all measured values three times the standard deviation, you can identified the RSSI measurement value is the gross error data exist, need to eliminate. The Pauta is used to eliminate gross error in the process of measurement data, need to complete the following steps:

a. $\operatorname{RSS}_{1 n}, \quad R S S I_{2 n} \ldots . . R S S I_{m n}$ is the intensity value of $\mathrm{N}$ node for $m$ times

measurement, take the average as $\mu, \mu=\frac{R S S I_{1 n}+R S S I_{2 n}+\ldots+R S S I_{m n}}{\mathrm{~m}}$

b. Calculation Standard deviation, $\sigma=\sqrt{\frac{1}{m} \sum_{i=1}^{m}\left(R S S I_{m n}-\mu\right)^{2}}$

c. Traverse $R S S I_{1}, R_{S S I_{2 n}} \ldots \ldots R S I_{m n}$, if it is satisfied $\left|R S S I_{m n}-\mu\right|>3 \sigma$, that the data is gross error, should be removed.

(2) Random error processing

After eliminating the gross error, the Kalman filter method is selected to handle the measured data. The principle of Kalman filter is as shown in style (1), assume that the current state of the system is $k$, if the established mathematical model is used to predict the state of the whole system, using this model, the most ideal value of the system in $k$ state is easy to get from $k-1$ state. $X(k \mid k-1)$ is the results obtained based on above state of the system. $A$ and $B$ are the parameters of the system in advance. $X(k-1 \mid k-1)$ is the optimal value of a state in the system. $U(k)$ is control variables for the current state of the system, $P(k \mid k-1)$ is the covariance of $X(k \mid k-1)$. $I$ is a unit matrix, $H$ is the parameter for the measurement system, $K_{g}$ is the Kalman gain of the system, $R$ and $Q$ is respectively the noise covariance in the measurement process, $Z(k)$ is the measured value at $k$ time. 


$$
\left\{\begin{array}{l}
X(k \mid k-1)=A X(k-1 \mid k-1)+B U(k) \\
P(k \mid k-1)=A P(k-1 \mid k-1) A^{T}+Q \\
X(k \mid k)=X(k-1 \mid k-1)+K_{g}(k)(Z(k)-H X(k \mid k-1)) \\
K_{g}(k)=P(k \mid k-1) H^{T} /\left(H P(k \mid k-1) H^{T}+R\right) \\
P(k \mid k)=\left(1-K_{g}(k) H\right) P(k \mid k-1)
\end{array}\right.
$$

In actual position environment, the receiver can scan RSSI of all wireless node for the current moment in a certain coordinate, but the value between measured and true is still exists a certain deviation. In most cases, it can be classified as Gauss white noise, which has no relation with time and obeys Gauss distribution. Therefore, we can get two RSSI values: measurement and empirical value according to the established positioning system model. The optimal RSSI value of the specific position can be calculated by using these two values in combination with their respective noise characteristics. RSSI of the systems should be exactly the same all time assuming that the receiving end coerdinates are unchanged, so the value of $A$ is 1 . Because the control quantity does notyexist in this location system, $U(k)$ is 0 , the estimate value of system or the current time is:

$$
\overline{R S S I}(k \mid k-1)=\operatorname{RSSI}(k-1 \mid k-1)
$$

$\operatorname{RSSI}(k-1 \mid k-1)$ is optimal value of system for $k-1$ time, $\overline{\operatorname{RSSI}}(k \mid k-1)$ is estimated result of system for the current time. According to the actual measured value and estimated value of RSSI, the best estination result of the current time is $\operatorname{RSSI}(k \mid k)$ by calculated. The value of measuremen (system parameter $H$ is 1 , get the following formula.

$$
\left.R S S I(k \mid k)=\overline{R S S I}(k \mid k-1)+R_{g} k\right)(R S S I(k)-\overline{R S S I}(k \mid k-1))
$$

In order to make the Kalman filter can realize the cycle calculation until the signal strength data processing is oyer, need to update the covariance of the $\operatorname{RSSI}(k \mid k)$, because the filtering model is a single model system, the covariance of $k$ time is:

$$
\begin{aligned}
& P(k \mid k)=\left(1-K_{g}(k)\right) P(k \mid k-1) \\
& \text { 2.2. Influence of Path Loss Index on Distance }
\end{aligned}
$$

\subsection{Influence of Path Loss Index on Distance}

In general, there is a certain error between the field strength value obtained by the experiment and the real value, according to the empirical model, the conversion of the field strength of the wireless AP node which is read from the receiving end to the distance will bring greate deviation to the positioning system. So it is necessary to modify the path loss model, this paper introduces the random variable $X_{\sigma}$, which will affect the random error as far as possible to minimize. The logarithmic attenuation model based on path loss is shown in the following formula:

$$
R S S I=R S S I_{0}-10 \gamma \log \left(\frac{d}{d_{0}}\right)+X_{\sigma}
$$

The error of path loss index will bring bigger error to the estimate distance between the receiver and the transmitter, ignoring the effects of noise, the following formula can be obtained.

$$
d=d_{0} \times 10^{\frac{R S S I_{0}-R S S I}{10 \gamma}}
$$

$\operatorname{RSSI}_{0}-\mathrm{RSSI}$ is said to $\Delta R$, so relative error $\delta$ is:

$$
\delta=\left|\frac{d-\hat{d}}{d}\right|=\left|1-10^{\frac{\Delta R}{10^{\gamma} \gamma}} \frac{\Delta R}{10^{\gamma}}\right|
$$


$\hat{d}, \hat{\gamma}$ is respectively gamma measurement value.

$$
\begin{gathered}
\frac{1}{\hat{\gamma}}-\frac{1}{\gamma}=\frac{\gamma-\hat{\gamma}}{\hat{\gamma} \times \gamma} \approx \frac{\Delta \gamma}{\gamma^{2}} \\
\delta=\left|1-10^{\frac{\Delta R}{10^{\gamma}}-\frac{\Delta R}{10^{\gamma}}}\right|=\left|1-10^{\frac{\Delta R \Delta \gamma}{10 \gamma^{2}}}\right|
\end{gathered}
$$

Generally take the average of $\gamma$, it is assumed that the orientation environment information can be reflected in 3.60 in this paper.

\section{Evaluation Function of Positioning System}

In the positioning algorithm based on distance measurement, the space distance intersection method is generally used to determine the coordinates of the nodes to be measured. Under the ideal conditions for the free space model respectively to more than three reference nodes as the center, to each reference node to be measured node distance as the radius of the circle intersect in one point, coordmates of the point is location of the node is to be measured, as shown in Figure.1.

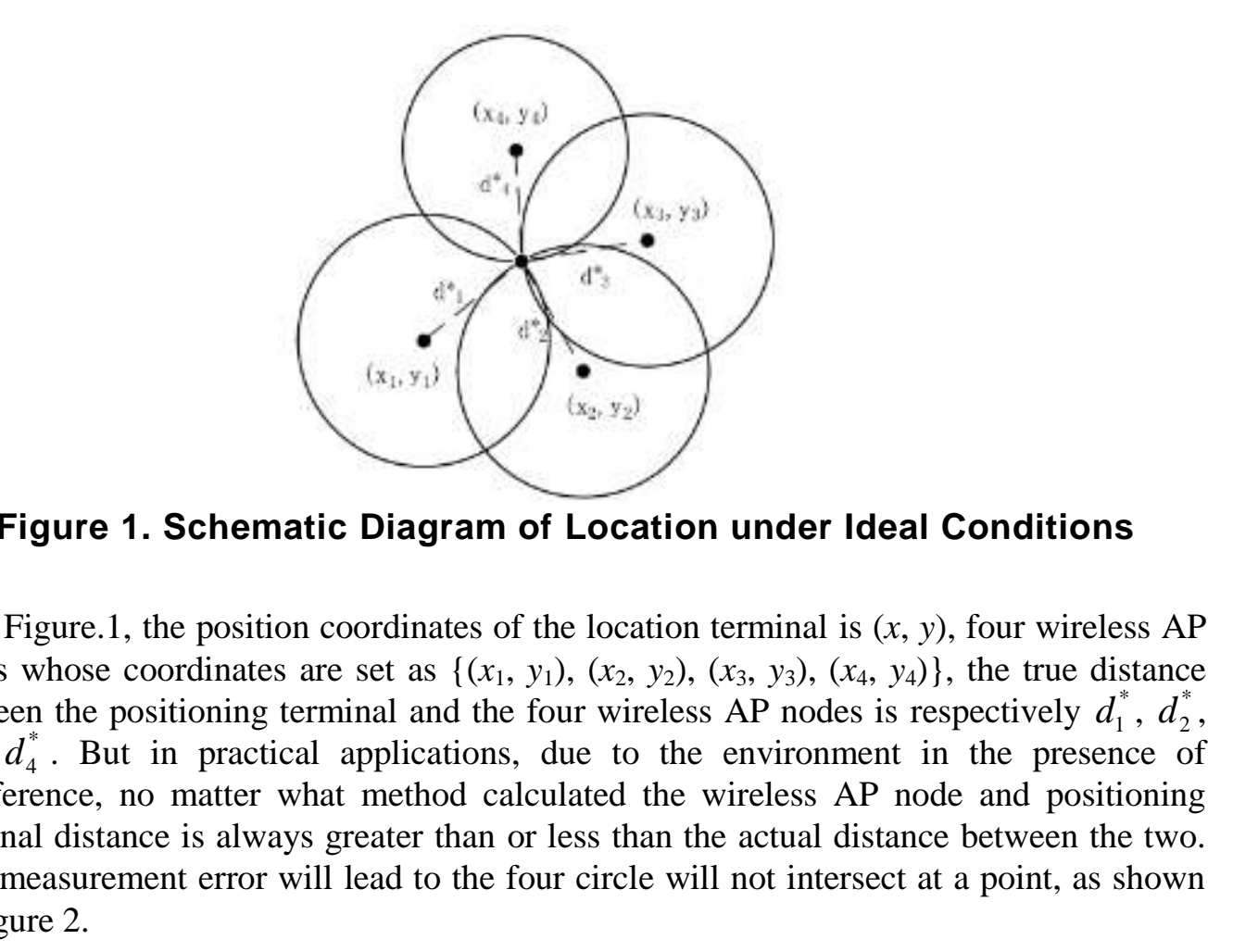




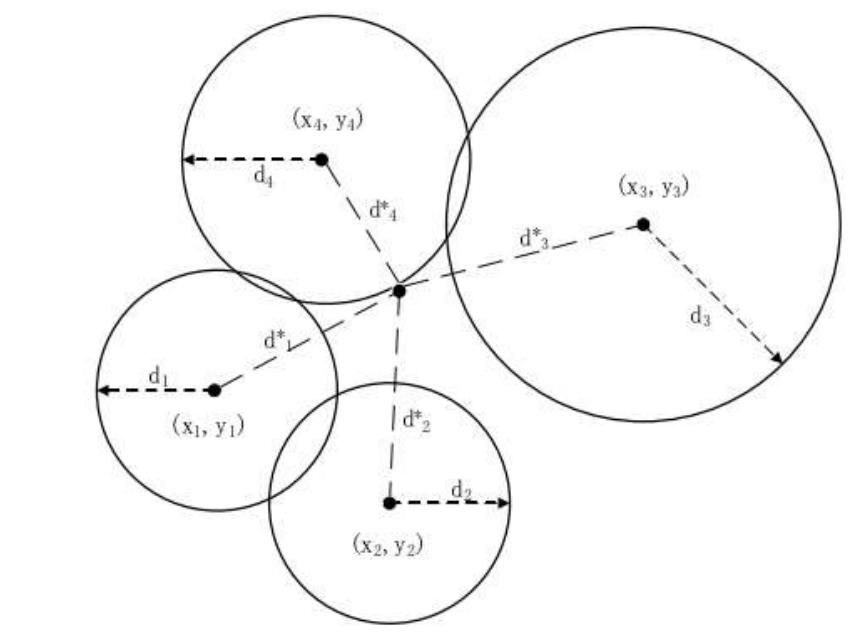

Figure 2. Schematic Diagram of Location in Realit

Assuming that in Figure 2, the coordinates of the intersection point between the shortest distance line segments for positioning terminal to the four borders round and positioning circle is respectively $\left\{\left(a_{1}, b_{1}\right),\left(a_{2}, b_{2}\right),\left(a_{3}, b_{3}\right),\left(a_{4}, b_{4}\right)\right\}$, the radius of the four circles is $d_{1}, d_{2}, d_{3}, d_{4}$, they are actually the estimated distance for positioning terminal to various wireless AP node. Ideally, the coordinate mapping and positioning terminal should be made one. The sum of the minimurn distance from the positioning terminal to all the boundary circles is the sum of the distances from the point to the mapping points. The following formula can be obtained.

$$
f(x, y)=\left|d_{1}^{*}-d_{1}\right|+d_{2}^{*}-d_{2}|+| d_{3}^{*}-d_{3}|+| d_{4}^{*}-d_{4} \mid
$$

When there are $n$ wireless AP hodes:

$$
\begin{aligned}
& \left.f(x, y)=\mid d_{1}\right)-d_{1}|+| d_{2}^{*}-d_{2}|+\ldots+| d_{n}^{*}-d_{n} \mid \\
& a_{i}=\sqrt{\left(x-x_{i}\right)^{2}+\left(y-y_{i}\right)^{2}}, i=1,2, \ldots n
\end{aligned}
$$

According to the above theory, this paper will put $f(\mathrm{x}, \mathrm{y})$ function as the system's positioning evaluation function, to determine the results of the positioning. The traditional positioning system is open loop; positioning evaluation function is used to make the positioning system to achieve a closed loop. In view of the problem of large amount of calculation of traverse coordinate point, the initial positioning and the middle position are directly solved by the least square method in this paper, and then use the location evaluation function to test the accuracy of the positioning results, then according to the test results to correct path loss index for repositioning, in order to improve the positioning accuracy of the system.

\section{Optimal Positioning Algorithm}

\subsection{Primary Positioning based on RSSI}

For the initial location of the system, need to make full use of the Wi-Fi network has information, select the larger signal strength of AP point, and given the specific value of the path loss index, the average path loss index is 4 . When the positioning end is added to the WiFi network, the signal strength value of each wireless AP point is scanned in the communication network. Then the signal strength value obtained by measurement is in 
accordance with Pauta test and Kalman filter, after filtering the RSSI sampled data, the signal intensity values of all wireless AP nodes are sorted, and select strong signal strength of the wireless AP point for the initial positioning, the initial positioning of the process is shown in Figure 3.

\section{Figure 3. Flow Chart of the Initial Positioning}

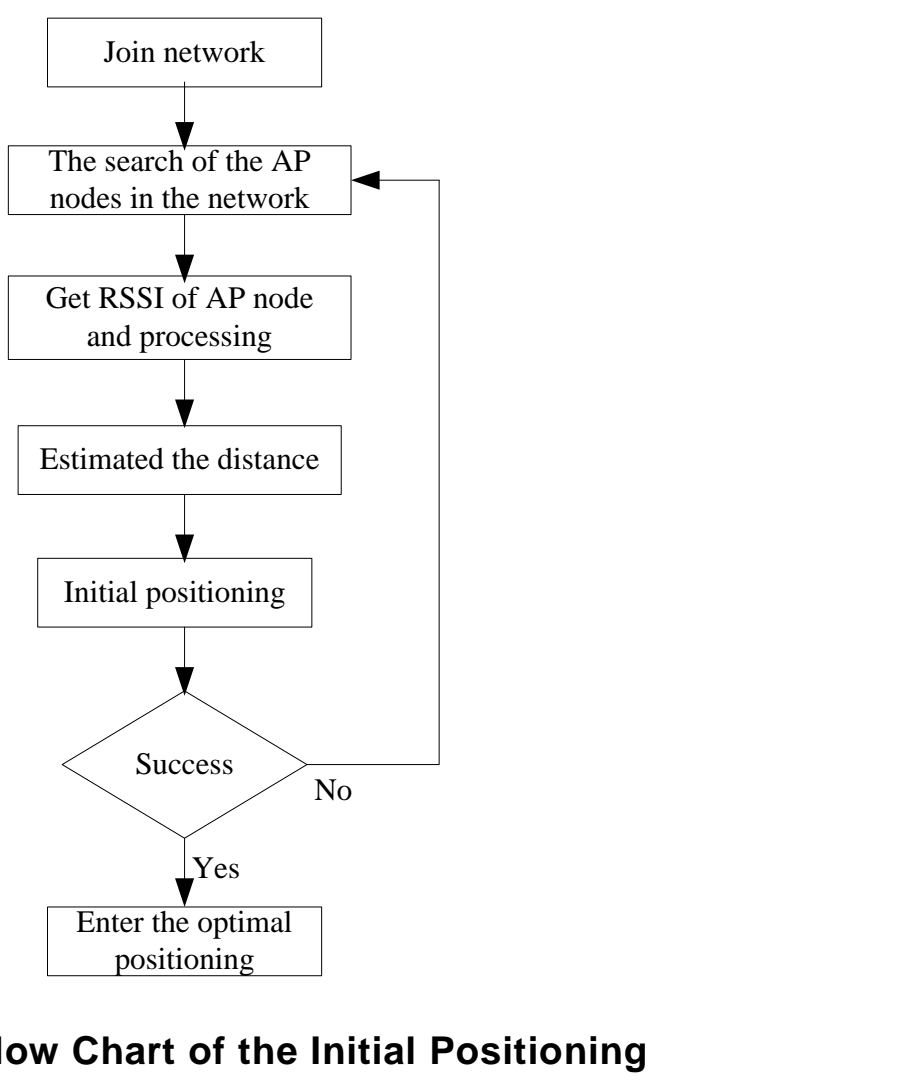

In the initial positioning the RSSI obtained will be in accordance with the size of the sort, select the strong point of the positioning of the signal, in general, the signal strength of the AP node below -70dBm oes not have value need to.

The method of least square method is used to solve the initial location, path loss index is $\gamma_{1}^{k}=\gamma_{2}^{k}=\ldots-\gamma_{n}^{k}=\bar{\gamma} \quad \gamma$ an empirical value in the current location environment, $k$ is 0 , calculate the distance, and then the system for positioning, to determine whether the positioning of success, if the positioning is successful, then enter the search for optimal positioning, or re acess to wireless AP nodes.

\section{.2. Optimization Update of Path Loss Index}

A ter the completion of the initial position based on the signal receiving strength, according to the results of the initial positioning to determine whether enter the optimal positioning. Because the prior information of the location environment is unknown and is easy to be affected by the movement of the object and people, the path loss exponent in different location environment is shown in Table 1. 


\section{Table 1. Path Loss Exponent in Different Location Environment}

\begin{tabular}{|c|c|}
\hline Location environment & Path loss index $\gamma$ \\
\hline Downtown area & $3.7-6.5$ \\
\hline Office & $2-6$ \\
\hline Free space & 2 \\
\hline Outdoors & 2.8 \\
\hline
\end{tabular}

Assignment optimization was carried out on the path loss index as follows; select the best path loss index $\gamma$. The main process is as follows:

1) According to the results of the initial positioning of each wireless AP node corresponding to the difference $\left|\sqrt{\left(x-x_{i}\right)^{2}+\left(y-y_{i}\right)^{2}}-d_{i}\right|$, in descending order, select the first $\mathrm{n} / 2$ optimization calculation, $\gamma_{\frac{n+1}{2}}^{k}=\gamma_{\frac{n+2}{2}}^{k}=\ldots=\gamma_{n}^{k}=\bar{\gamma}$, remain unchanged.

2) From Table 1 shows that the value of the path loss exponent in the common location environment is between 2 6.5, from this we can know $\gamma_{1_{-} \text {min }}^{k}=\gamma_{2_{-} \min }^{k}=\ldots=\gamma_{n / 2 \_ \text {min }}^{k}=\gamma_{\text {min }}=2 \quad, \quad \gamma_{1_{-} \max }^{k}=\gamma_{2}^{k}$ man $=\ldots=\gamma_{n / 2 \text { max }}^{k}=\gamma_{\max }=6.5$, $s_{1}=s_{2}=\ldots=s_{n / 2}=\left(\gamma_{\max }-\gamma_{\min }\right) / \eta, \eta$ is a positive integer, $k$ is searching times, in the initial seasonal, $k=1, j=1$.

3) Different processing strategies are adopted according to the $k$ value. When $k=1$, $\gamma_{m}^{k}=\bar{\gamma}_{m}^{k}(m=1,2, \ldots, j-1), \bar{\gamma}_{m}^{k}$ has been optimized for the $m$ bit, at the same time, $\gamma_{j+1}^{k}=\gamma_{j+2}^{k}=\ldots=\gamma_{n / 2}^{k}=\bar{\gamma}$. When $k>1, \gamma_{m}^{k}=\gamma_{m}^{k}(m \leqslant 1,2, \ldots, j-1, j+1, \ldots, n / 2)$.

4) $\gamma_{j}^{k}$ is $\alpha, \alpha+s, \alpha+2 s, \ldots, \alpha+\eta s$ respectively, $\gamma^{k}(t)=\alpha+t s, t=0,1,2, \ldots, \eta$.

5) Acquisition range set based on $X^{k}$, using the least square method to calculate the coordinate value, put the coordinate value through the positioning evaluation formula (12).

6) Selec the minimum of evaluation formula corresponding $\gamma_{j}^{k}, \gamma_{j}^{k}$ is current optimization value for bit. The optimal path loss index obtained is used to find the optimal $\gamma_{j+1}^{k}$, if $j=\mathrm{n} / 2$, complete a rough search, otherwise, $j=j+1$, repeat 3) $\sim$ ) step.

7) Make $k=1, j=1, \gamma_{j_{-} \min }^{k}=\max \left(2, \bar{\gamma}_{j}^{k-1}-s\right), \gamma_{j_{-} \max }^{k}=\min \left(\bar{\gamma}_{j}^{k-1}+s, 6.5\right)$, to reset $s_{\mathrm{j}}$, make $s_{j}=\left(x_{j_{-} \text {max }}^{k}-\gamma_{j_{-} \text {min }}^{k}\right) / \eta$, repeat 2) 6) step. When the maximum value of $s_{\mathrm{j}}$ is less than the set threshold, the optimization is done.

\subsection{Final Location based on RSSI}

Using the above process to obtain a set of optimal path loss index, compute the distance from location to the selected wireless AP node, then the weighted centric localization algorithm is used to carry on the final positioning. According to the characteristics of $\mathrm{WiFi}$ positioning, the closer distance wireless AP node, the greater its influence. In this paper, $1 / \mathrm{d}_{\mathrm{i}}$ is selected as the weighting factor of the positioning system. The calculation formula of the position terminal position is as follows:

$$
x=\frac{x_{1}}{d_{1}}+\frac{x_{2}}{d_{2}}+\ldots+\frac{x_{n}}{d_{n}} / \frac{1}{d_{1}}+\frac{1}{d_{2}}+\ldots+\frac{1}{d_{n}}
$$




$$
y=\frac{y_{1}}{d_{1}}+\frac{y_{2}}{d_{2}}+\ldots+\frac{y_{n}}{d_{n}} / \frac{1}{d_{1}}+\frac{1}{d_{2}}+\ldots+\frac{1}{d_{n}}
$$

In the above formula, $x_{\mathrm{n}}, y_{\mathrm{n}}$ are coordinates for wireless AP node, $d_{\mathrm{n}}$ is the estimated distance calculated by the path loss exponent.

\section{Experiment and Result Analysis}

In order to verify the positioning performance of the optimization algorithm in the indoor environment, the laboratory experiment was conducted in the laboratory, which was complicated by the indoor environment, more people and often moving around. Computer and other electronic equipment in the laboratory are more, the interference source is more, and the local map of the scene is shown in Figure 4.

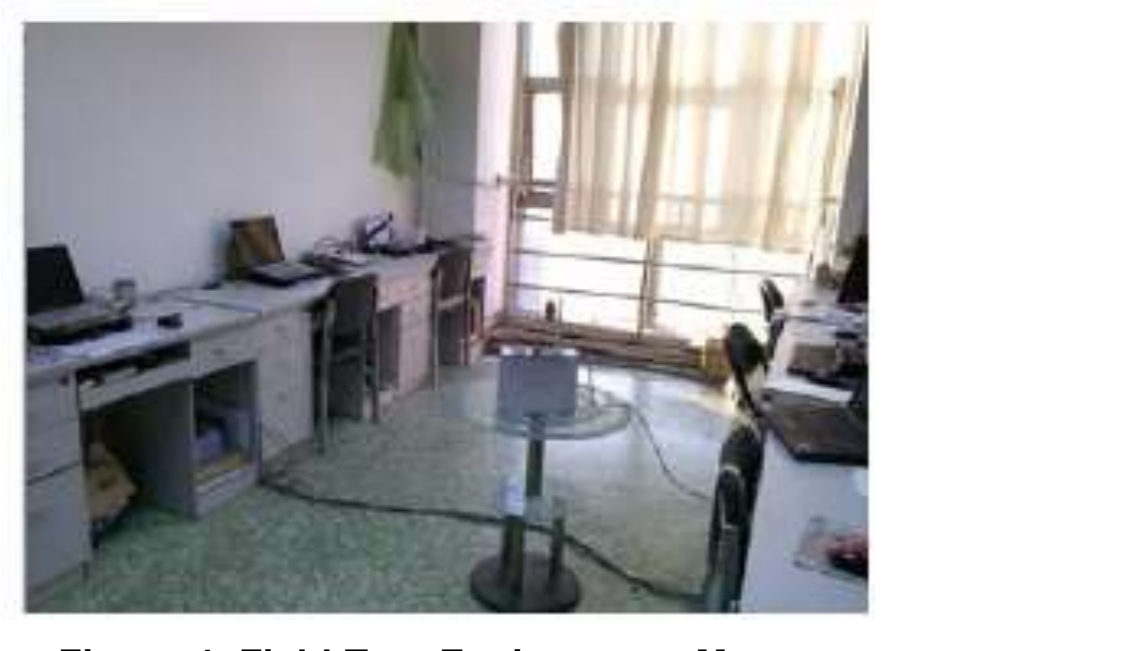

Figure 4 Field Tèst Environment Map

The laboratory is 7.50 meters long and 6.50 meters wide, deployed 4 known locations of wireless AP nodês. The layout is shown in Figure 5, establish the right angle coordinate system based on the location of AP), the coordinates of the 4 wireless AP nodes are $\mathrm{AP} 1(0,0), \mathrm{AP} 2(6.00,0), \quad \mathrm{AP} 3(6.00,5.50), \mathrm{AP} 4(0,5.50), 8$ test points were selected. Each test point is measured in advance to its true location, number is $1 \sim 8$. Network layout and test point location rap are as shown in Figure 5.

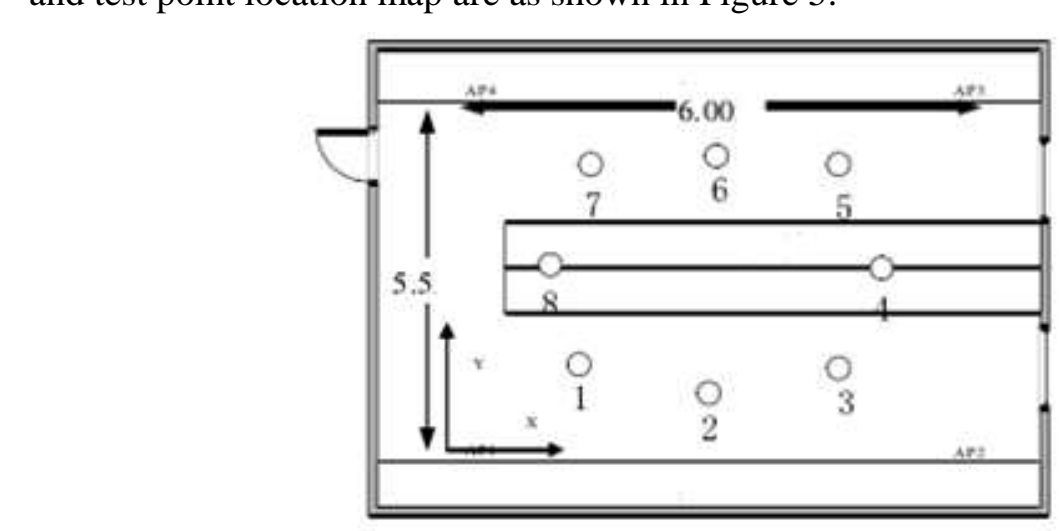

Figure 5. Network Layout and Test Point Location Map

In experiment $d_{0}=1.00 \mathrm{~m}, \eta=5, R_{0}$ is the average value for 500 times measured at $d_{0}$, experimental measurement $R S S I_{0}=-18 \mathrm{dBm}$. The threshold setting for the stopped optimal 
location is 0.10 . After traversing, the sum of 8 test point positioning error is minimum when $\gamma=3.6$, so the path loss index is fixed 3.6 in the non optimal location algorithm.

Field experiments are carried out according to the above mentioned parameters, the positioning terminal is moved to 8 known locations of the test points, the signal strength of four wireless AP nodes is measured at each test point 20 times. The height of the above 8 measurement points is 0.75 meters. After pull up to the sampled data in Pauta inspection and Calman filter to locate, the experimental results are shown in Figure 6.

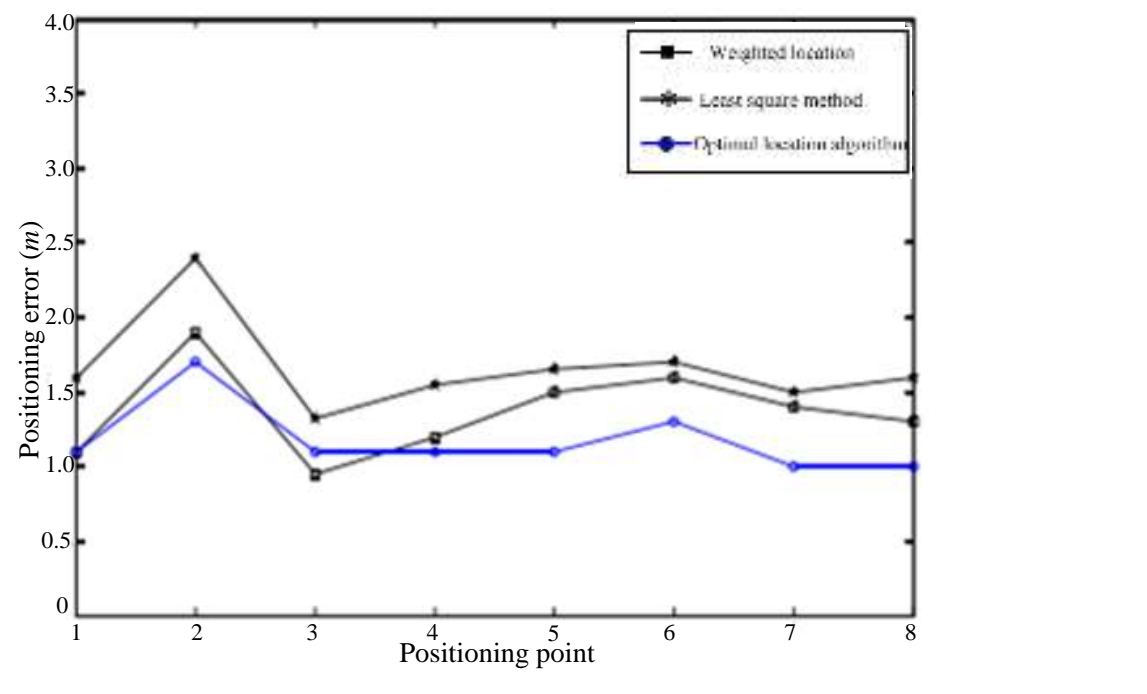

Figure 6. Eight Test Points and Corresponding Positioning Error

Figure 6 shows that the optima Rocation algorithm is better than the non optimization algorithm; the average error of the 8 test points is 1.12 meters, the average location error of the weighted centroid localization algonithm is 1.36 meters, the average location error of the least square method is 1.66 meters. In contrast to the open environment test, the average location error of the optimal algorithm is the least; positioning error has only increased by $0.27 \mathrm{~m}$, the positioning errors of other two kinds of positioning algorithm are increased by about 0.40 meters, when the location environment changes, the optimal location algorithm shows a high stability and adaptability.

\section{Conclusion}

At present, the demand for positioning and navigation is gradually increasing and the outdoor positioning and navigation is relatively mature, but in the complex indoor environment, such as hospitals, supermarkets, subway stations and other indoor environment, the positioning system is still unable to meet the needs of people. Because of the complexity of indoor location environment, it is difficult to determine the path loss index, in view of the actual demand of the positioning system, this paper puts forward a method to find the optimal location, by introducing the positioning evaluation function, and the path loss index is optimized to improve the positioning accuracy, it has certain popularization and application value.

\section{Acknowledgements}

This work was supported by the Natural Science Foundation for Young Scientists of Henan Polytechnic University under Grant Q2016-2A, and Key Laboratory of control engineering of Henan Province, Henan Polytechnic University. 


\section{References}

[1] Prasithsangaree P, Krishnamurthy P and Chrysanthis P K, Editors. On indoor position location with wireless LANs. Proceedings of the 13th IEEE International Symposium on Personal, Indoor and obile Radio Communications, (2002) July 10-13; Wuhan, China.

[2] Sugano M, Kawazoe T and Ohta Y. Target, 4, 12 (2006).

[3] H. Li, J. Meng,Y. Shi, J.X. Cai. A Statistical Channel Model for 2.4GHz Radio Propagation in Ruins Environment. Revista de la Facultad de Ingeniería, Vol.31, No.6, (2016), pp.53-63.

[4] Zaruba G V, Huber M and Kamangar F A. Wireless networks, 2, 3 (2007).

[5] Koo J and Cha H. Communications Letters, 2, 15 (2011).

[6] So J, Lee J Y. International Journal of Software Engineering and Its Applications, 3, 7 (2013).

[7] Boonsriwai S, Apavatjrut A, Editors. Indoor WIFI localization on mobile devices. Proceedings of the 10th International Conference on Electrical Engineering, Computer, Telecommunications and Information Technology, (2014) October 21-23; Beijing, China.

[8] Altintas B and Serif T, Editors. Indoor location detection with a RSSI-based short term memory technique. 2012 IEEE International Conference on Pervasive Computing and Communications Workshops, (2012) June 6-8; Kyoto, Japan.

[9] Zhongliang D, Yanpei Y and Xie Y. Communications, China, 3, 10 (2013).
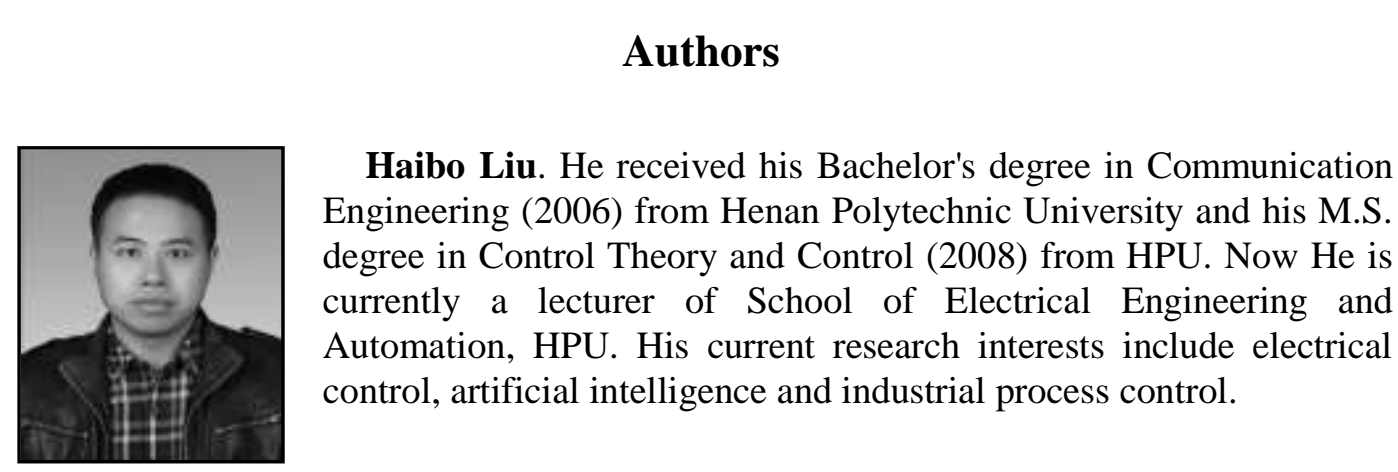

Haibo Liu. He received his Bachelor's degree in Communication Engineering (2006) from Henan Polytechnic University and his M.S. degree in Control Theory and Control (2008) from HPU. Now He is currently a lecturer of School of Electrical Engineering and Automation, HPU. "His current research interests include electrical control, artificial intelligence and udustrial process control.

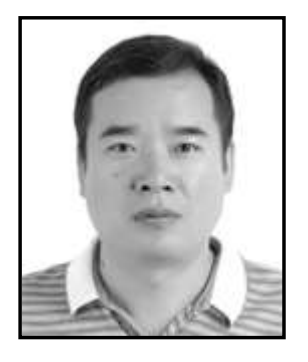

Yujie Dong. He received his Bachelor's degree in Computer Science and Technology (2000) from Henan Polytechnic University and his M.S. degree in Control Theory and Control (2006) from HPU. - Now He is currently an assistant professor of School of Electrical Engineering and Automation, HPU. His current research interests include electrical control, artificial intelligence and industrial process control.

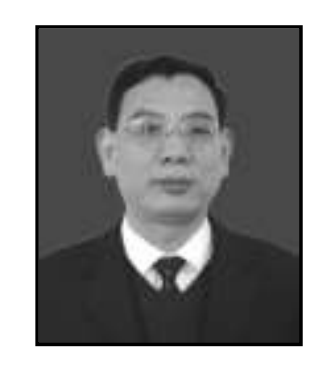

Kongle Ai. He received his $\mathrm{PhD}$ in Electrical Engineering from University of Stellenbosch, South Africa in 2006. He joined the Henan Polytechnic University from 1987 to now. He is currently a Professor at School of Electrical engineering and Automation, Henan polytechnic University, China. His research interests include multiphase $\mathrm{AC}$ machine control and power system filter. 